\title{
Français et créole : contact de langues aux Antilles
}

\author{
Iskra Iskrova \\ Département de Français \\ Université Indiana à Bloomington \\ iiskrova@indiana.edu
}

\section{Introduction}

Les Antilles sont une zone de contact de langues permanent et continu depuis le $17^{\mathrm{e}}$ siècle. Le premier contact de langues entre le français colonial et les langues africaines apportées par les esclaves fut à l'origine de la formation des créoles que nous connaissons aujourd'hui. De nos jours, créole et français sont, à leur tour, en contact. L'analyse du créole a longuement alimenté la curiosité des chercheurs, y compris les influences souvent considérées néfastes du français sur le créole. En revanche, il y a une lacune d'analyses examinant l'empreinte du créole sur le français aux Antilles. Il est indéniable que le français standard, variété de prestige, est une source d'emprunts pour les Guadeloupéens bilingues. Cependant, on ne peut ignorer le fait qu'un grand nombre de Guadeloupéens ont grandi dans des foyers monolingues et n'ont appris le français qu'en milieu scolaire. Pour ces locuteurs le français est une langue seconde. On peut s'attendre que leur langue première, le créole, a déteint sur le français. On n'a pas de chiffre officiel pour les monolingues à la naissance. Cependant, si l'on faisait des enquêtes, le nombre pourrait être plus élevé qu'on ne l'imagine. Étant donné que le français est demeuré langue seconde pendant longtemps, il est parfaitement plausible que le créole façonne à sa manière le domaine du français aux Antilles

En l'absence d'études préalables sur le français antillais, certaines idées exposées ici sont des hypothèses de travail qui permettront à terme de clarifier le rapport entre le français antillais et le créole. Cet exposé présente d'abord les variétés linguistiques en présence, pour ensuite explorer certaines caractéristiques communes du créole guadeloupéen et du français antillais. La fin de l'exposé se concentre sur l'intonation. Le domaine de la prononciation et de l'intonation représente, en effet, la sphère de la langue dans laquelle les influences du créole sur le français sont irréfutables.

\section{Variétés linguistiques en Guadeloupe}

Trois variétés de langue font partie du paysage linguistique quotidien en Guadeloupe. Ces langues sont organisées sur un continuum qui va du créole au français standard, en passant par le créole francisé et le français créolisé.

Le français standard et les variétés de français de la métropole sont l'apanage de la catégorie appelée localement les métros, à savoir les fonctionnaires, des cadres venus de France, les militaires et les différents métropolitains qui peuplent l'île. Leur nombre serait entre 7300 (selon les données de 2004 sur la fiche pays dans Ethnologue) et 9200 (selon les données courantes de Joshua Project) sur une population de 407719 habitants dans l'archipel Guadeloupe au $1^{\text {er }}$ janvier 2009 (INSEE). Par commodité je regrouperai ces variétés venues de la métropole sous l'étiquette français hexagonal (FH). Le français hexagonal ne constitue pas une catégorie homogène. Ce terme réfère à un ensemble de parlers provenant de la métropole, incluant le français standard aussi bien que des variétés régionales de France. Ces variétés sont en usage dans une catégorie sociale mouvante et changeante, étant donné les contrats de trois ans dans la fonction publique et la nature généralement temporaire du séjour des métropolitains en Guadeloupe. Cette variété, la plus proche du standard, est parlée par une minorité de la population de l'île. Parmi les locuteurs du français hexagonal on doit inclure aussi les Guadeloupéens établis en 
métropole ou nés en métropole, ceux appelés de l'autre côté de l'Atlantique, les négropolitains. Quoique rares, certains Guadeloupéens de métropole reviennent vivre en Guadeloupe. Ils ne parlent pas nécessairement le créole et leur première langue est le français hexagonal. Le français standard constitue la langue cible dans l'éducation et dans les salles de classe en Guadeloupe. Le contact des Guadeloupéens avec le français standard ne se limite pas uniquement aux interactions directes, puisque le français standard est largement diffusé sur les médias : radio, télévision, aussi bien que la presse en forme écrite.

Outre le français hexagonal, dont le représentant le plus proéminent serait le français standard, il existe une variété locale de français, le français antillais (FA). Le français antillais, qui constitue le principal objet de cet exposé, présente bien de traits endogènes et il est identifié par les Français de la métropole comme différent des français de l'Hexagone. Même si l'éducation nationale se donne pour objectif d'enseigner le français standard en Guadeloupe, le fait est que chaque Guadeloupéen bilingue parle le français antillais. Seules les élites locales et des Guadeloupéens qui ont longuement séjourné en métropole tendent à parler un français proche du français standard.

Le français antillais constitue une des nombreuses variétés de français d'Amérique. Ce parler se distingue des variétés hexagonales, dont il dérive, par sa longue présence aux Antilles. La souche du français antillais serait le français colonial, la koinè en usage parmi les colons qui se sont établis aux îles. Les caractéristiques du français antillais sont à rechercher dans le français colonial et dans son contact continu avec le créole. C'est un fait que la majorité de la population guadeloupéenne est aujourd'hui bilingue, même si ces mêmes locuteurs sont issus de familles monolingues. Mon hypothèse est que dans le processus d'apprentissage de la langue seconde, qui est le français, les créolophones de naissance ont eu l'opportunité de transférer des traits de créole dans la variété de français locale. Et de ce fait, certaines caractéristiques du français antillais ne sauraient être comprises sans référence au créole. Il est essentiel de se méfier du piège qui consiste à croire que le français antillais n'est que du français créolisé. Le français créolisé devrait constituer une autre variété sur le continuum, mais ce n'est pas la seule nature du français aux Antilles. En d'autres termes, le français antillais n'est pas du français standard mal parlé.

Enfin, le créole guadeloupéen (CG) demeure le principal vernaculaire de l'île. Il n'est pas de Guadeloupéen non négropolitain qui ne parle pas ou ne comprend pas le créole. Les attitudes linguistiques déterminant le choix de la langue qu'on parle dans différentes situations de communication sont extrêmement intriquées et ne peuvent être détaillées ici. Il est cependant intéressant de remarquer que même ceux qui choisissent délibérément de ne pas s'exprimer en créole, ont une compétence incontestée en créole. La typologie des locuteurs en fonction de leur attitude par rapport au français et le créole serait trop fastidieuse ici, mais je voudrais en indiquer quelques catégories pertinentes pour mon propos ici. Contrairement à ce que l'on pourrait croire, on trouve encore en Guadeloupe des monolingues. Ce groupe est souvent limité aux personnes âgées, mais il comprend également des ouvriers dans leur cinquantaine qui ont échappé aux circuits de la scolarisation obligatoire. Il existe un très grand nombre de Guadeloupéens, d'un âge moyen, pour lesquels le français n'est qu'une langue seconde. Ces locuteurs ne furent exposés au français qu'en milieu scolaire et ont été élevés dans des foyers exclusivement créolophones. Malheureusement, aucune enquête n'a été menée et il est impossible de donner des valeurs chiffrées pour cette population, dont la taille n'est pas négligeable.

\section{Traits partagés}

Il existe quelques traits communs au créole et au français qui ne peuvent pas être liés au français hexagonal et qui suggèrent que le créole a laissé son empreinte sur le français parlé aux Antilles. Loin de présenter une grammaire comparée du français antillais, seules quelques caractéristiques communes au créole et au français antillais seront mentionnées. Le français antillais a hérité, d'une part, d'un fonds lexical provenant des langues indigènes de la Caraïbe, telles que le caribe et l'arawak. Ceci est le cas de noms de la flore et faune endogènes à cette région et qui sont inconnus, ou peu connus en métropole. Ainsi, localement préfère-t-on le terme de maracudja (prononcer [marakudza]) à « fruit de la passion ». Il est intéressant de remarquer que des locuteurs basilectaux ou quasi-monolingues croient que le terme maracudja est français, puisque c'est le terme ordinaire en français antillais, qui est aussi communément 
utilisé par les métropolitains résidant sur l'île. Ce terme qu'on retrouve également en créole fait partie intégrante du français antillais. Le créole a d'autres termes, tels que ponm lyann qui ne sont pas rentrés dans le fonds lexical du français antillais. Le vocabulaire "autochtone», celui provenant des langues précolombiennes de la Caraïbe, est vraisemblablement rentré simultanément en français colonial et en créole. En plus du vocabulaire d'origine précolombienne, le français antillais comprend un grand nombre d'acceptions vieillies et considérées obsolètes en français hexagonal. Par exemple, linge signifie " vêtements » aux Antilles, à l'instar du créole. Les maisons à étage sont désignées de maison haute, qui est une réplique de kaz wòt en Guadeloupe et kay wòt en Haiti. Le scénario le plus plausible est que les termes et acceptions se sont maintenus dans l'évolution du français colonial au français antillais et ils ont été transmis du français colonial au créole. Ces termes ne sont donc pas des emprunts directs au créole. Cependant la question se pose de savoir pourquoi ces termes vieillis par rapport au français standard se maintiennent en français antillais alors qu'au même moment le fonds lexical créole se retrouve fortement altéré par la présence du français. Cette situation nécessite un examen approfondi. En attendant, il me semble que le créole et le français antillais se soutiennent mutuellement pour maintenir des acceptions héritées du français colonial. Dans cette hypothèse, le créole jouerait un rôle d'agent de conservation du fonds lexical vieilli et obsolète en français antillais. Il est certainement plus facile pour ces termes de résister l'influence du français standard à deux.

Sur le plan syntaxique, le cas de la structure factitive est assez saillant. La structure factitive en français antillais se distingue nettement du français hexagonal. Celle-ci est manifestement liée à la structure créole, comme illustré dans les exemples en (1). La structure créole est par ailleurs commune aux créoles de la Caraïbe, et non une caractéristique essentiellement guadeloupéenne.

$$
\begin{array}{llllll}
\text { FH } & \text { j'a fait faire le devoir aux enfants. } & & \\
\text { CG } & \text { an te fè } & \text { se timoun la } & \text { ekri } & \text { devwa la. } \\
& \text { je } & \text { PASSÉ faire } & \text { les-enfants } & \text { écrire } & \text { le-devoir. }
\end{array}
$$

FA j'ai fait les enfants écrire le devoir.

La prononciation antillaise a besoin de faire l'objet d'une description détaillée. En attendant, je ne vais mentionner que le cas de [r]. L'affaiblissement des [r] français a conduit à leur articulation en tant que glides [w] dans le cas de wòch pour 'roche', voire leur disparition en créole comme dans bò pour 'bord'. Il semble que les [r] ont chuté en position finale, ils sont devenus [w] en position d'attaque suivi d'une voyelle arrondie, qui est par défaut une voyelle d'arrière, voir wòb, wòch, et wou pour 'robe', 'roche' et 'roue' respectivement, mais ils se sont maintenus en attaque suivie d'une voyelle d'avant comme dans rezen 'raisin' et lari 'rue', dans ce dernier la voyelle étant passée de [y] à [i] puisque le créole élimine les voyelles d'avant arrondies. Les [r] qui ont demeuré en créole ont aussi pour caractéristique d'être vélaires et non pas uvulaires comme en français. Ceci est vrai à la fois pour le créole et le français antillais. La chute des [r] n'a pas eu lieu de façon systématique en français antillais. En revanche, la réalisation [w] en position finale est devenue standard dans cette variété de français: la mèw 'la mer', pomme de tèw 'pomme de terre', bouilliw 'bouillir, Pyèw 'Pierre'. La réalisation en [w] bien que plus contrôlée et évitée à un certain degré chez les Antillais éduqués se retrouve parmi toutes les catégories sociales en Guadeloupe.

Enfin, l'intonation offre un cas d'étude intéressant. Les caractéristiques intonatives du créole guadeloupéen seront présentées dans la section qui suit. Ensuite quelques exemples en créole antillais vont mettre en évidence des ressemblances entre les deux systèmes.

\section{L'intonation en créole guadeloupéen}

Le système intonatif du créole guadeloupéen fut décrit selon un corpus étendu d'énoncés enregistrés auprès de locuteurs natifs. L'exposé se concentre ici uniquement sur les aspects de l'énoncé déclaratif. 


\subsection{Méthode}

L'analyse des patrons intonatifs dans l'énoncé déclaratif en créole guadeloupéen est basée sur un corpus de 1082 occurrences produites par quatre locuteurs, PD, MX, PW et DL. Trois des quatre locuteurs ont été élevés dans des familles monolingues et n'ont commencé à apprendre le français qu'en milieu scolaire. Le dernier locuteur a grandi dans un ménage francophone, mais utilisait le créole avec ses grands-parents et en tant que langue de jeu avec ses amis d'enfance. Les données provenant de ce participant se recoupent complètement avec les données des autres locuteurs et ne rentrent pas en contradiction avec le reste du corpus. Ces données furent donc intégrées dans l'analyse. Signalons aussi qu'une des participantes est quasi-monolingue. C'est une femme qui est toujours dans la vie active. Elle n'a pas poursuivi l'école au delà de la deuxième année (l'équivalent de CE2). Elle travaille dans un milieu essentiellement créolophone et elle vit seule sur la même propriété que sa mère qui est incontestablement monolingue. Sa compréhension du français est approximative et elle ne maîtrise point le français oral.

Pour une première description du système intonatif, il était essentiel de collecter des énoncés uniformes qui se prêtaient facilement à une analyse instrumentale. Afin d'obtenir des données homogènes, les participants ont construit des phrases 'écrites' en images, à l'instar des exemples en (2). De même, afin d'éviter les interruptions de la fréquence fondamentale (F0) les énoncés étaient construits avec des mots comprenant uniquement des sonantes. Seuls quelques rares mots lexicaux et les fonctifs font exception à cette règle. Il était donc non seulement essentiel de produire les mêmes énoncés, mais aussi d'utiliser le même vocabulaire, qui fut soigneusement sélectionné par mes soins. Pour cela, toute séance d'enregistrement commençait par une séquence d'apprentissage du vocabulaire qui correspondait à chaque image. Les énoncés étaient construits en sorte de contrôler le nombre de syllabes dans les différentes positions syntaxiques. Les énoncés du corpus vont de phrases très courtes du type SVO, à des énoncés complexes avec des relatives et des subordonnées, dont les exemples en (3) constituent un échantillon. Les phrases furent élicitées dans un dialogue sous forme de questions et réponses entre l'enquêteur et le locuteur natif. Chaque énoncé fut produit trois fois, dans des interactions séparées.

(2)

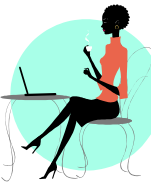

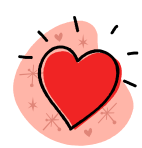

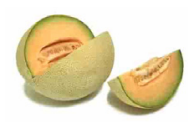

Enoncé correspondant: Lin enmé melon. « Lyne aime le melon. »
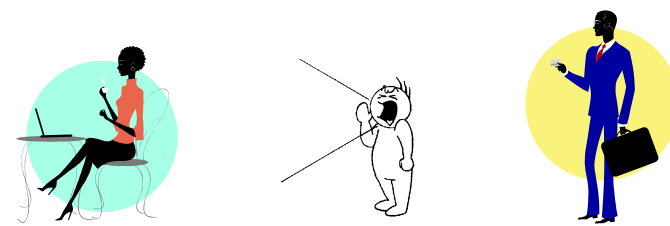

Enoncé correspondant: Lin hélé Orelyen. « Lyne appelle Aurélien. »

(3) a. Alen enmé Emilyèn.

« Alain aime/est amoureux d'Emilienne. »

b. Wonmyè la enmé manawa la.

« Le soiffard aime/est amoureux de la catin. »

c. Wonmyè la enmé manawa la ki rina.

« Le soiffard aime/est amoureux de la catin qui est radin. »

d. Wonmyè la mannyè on melon.

« Le soiffard a volé un melon. » 
e. Wonmyè la ki enmé wonm mannyè on boutèy wonm andan lolo la. « Le soiffard qui aime le rhum a volé une bouteille de rhum dans l'épicerie. »

f. $\quad$ Wonmyè la hélé lamayè lè i bwè wonm.

« Le soiffard a interpellé la mariée pendant qu'elle buvait du rhum. »

Les avantages d'une méthode imagée sont incontestables dans un lieu où il n'y a pas de convention orthographique standard et où la majorité des locuteurs ne savent pas lire et écrire leur langue. Cette méthode a eu le triple avantage de (a) faire produire les mêmes énoncés à tous les participants, (b) générer des énoncés verbaux quasi naturels, par opposition à des énoncés lus qui n’auraient pas été représentatifs du discours spontané, et (c) inclure dans l'étude des sujets monolingues et illettrés. Au passage soit dit, il n'y a toujours pas une orthographe standard en Guadeloupe. Qui plus est, la majorité des Guadeloupéens, qui lisent le français, ne savent pas lire et écrire le créole. Ces faits compliquent immensément toute tentative d'éliciter des données au moyen de supports écrits.

\subsection{Cadre théorique de l'analyse}

Les énoncés furent examinés avec le programme Praat et analysées dans le cadre théorique de la phonologie autosegmentale-métrique, qui puise ses principes dans le travail de Pierrehumbert (Pierrehumbert, 1980) sur l'anglais, puis fut étendue avec des travaux sur le japonais en collaboration avec Beckman (Beckman and Pierrehumbert, 1986, Pierrehumbert and Beckman, 1988). Ce modèle s'est avéré très productif pour rendre compte des systèmes intonatifs de langues appartenant à des groupes linguistiques différents. Le cadre théorique présuppose la hiérarchie des constituants prosodiques (Nespor and Vogel, 1986), ainsi que l'alignement des frontières de chaque constituant avec des frontières des constituants de niveau inférieur ou supérieur (Selkirk, 1980). La courbe intonative est décrite en termes de deux tons, haut $(\mathrm{H})$ et bas $(\mathrm{B})$. La description rend compte des moments pivot de la courbe intonative où elle change de direction et des transitions entre deux tons.

Le marquage de l'information prosodique dans l'énoncé s'organise à trois niveaux, qui sont le mot prosodique (MPr), le syntagme accentuel (SA) et le syntagme intonatif (SI), représentés dans leur organisation hiérarchique en (4). Le marquage intonatif s'opère entre MPr, SA et SI.

(4) Hiérarchie des constituants prosodiques en CG

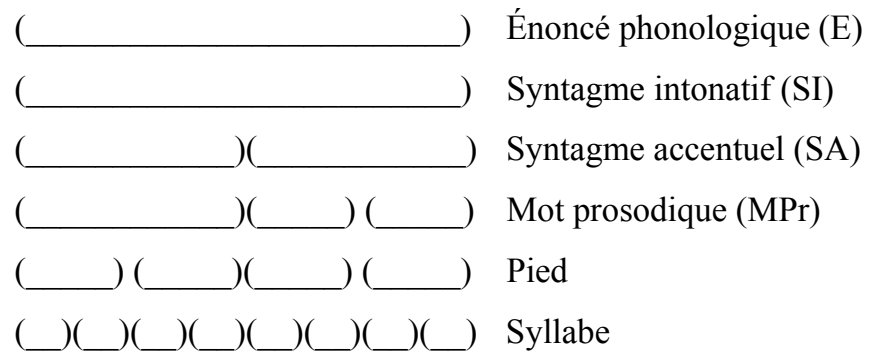

\subsection{Brève description du système intonatif du créole guadeloupéen}

Fig.1 schématise l'organisation des constituants prosodiques et l'organisation des tons intonatifs en créole guadeloupéen. Chaque mot prosodique reçoit un patron intonatif. Il ne s'agit cependant pas d'un accent de mot, ni de patron tonal lexicalisé, tels qu'on les trouve dans certaines langues africaines. La preuve en est que le même mot peut apparaître avec un patron tonal différent en fonction de sa position dans l'énoncé, ou plus précisément dans le syntagme accentuel. Il s'agit de tons intonatifs et non de tons lexicalement distinctifs. A cet égard, l'espace tonal (i.e. la succession des tons intonatifs) du créole 
guadeloupéen est beaucoup plus dense que l'espace tonal du français. Le syntagme accentuel comprend un patron en forme de chapeau, avec ascension et chute. Chaque SA contient deux mots prosodiques, dont le premier (MPr-1) reçoit la courbe montante, et le deuxième (MPr-2) reçoit la courbe descendante. La montée en MPr-1 est associée avec la syllabe finale de mot. Pour rendre compte de la transition qui s'opère toujours à l'intérieur de la même syllabe, le ton est transcrit comme $\mathrm{B}+\mathrm{H}$, i.e. une configuration bitonale qui se réalise à l'intérieur de la même syllabe. En revanche, le début du mot est toujours associé à un ton bas, transcrit B. Le début du MPr-2 est associé à un ton haut. Il est clair qu'il s'agit d'un ton haut en soi puisque ce ton peut se réaliser à une fréquence différente du ton haut du MPr précédent, tout comme il peut rester sur le même niveau, en formant un haut plateau. La différence est illustrée dans le schéma par la différence de réalisation entre le premier et le deuxième SA. Ce ton haut initial est suivi par un ton descendant $\mathrm{H}+\mathrm{B}$ qui est systématiquement associé à la pénultième. La syllabe finale demeure au niveau bas. La descente en pénultième position donne une forte impression de proéminence da la pénultième syllabe. Plusieurs SA se combinent dans un syntagme intonatif. La fin du SA peut être marqué par un ton haut (H-) ou un ton bas (B-). Le diacritique «-» représente l'association avec la frontière finale du domaine. Ce ton est associé au niveau du SA dans la hiérarchie proposée en (4), par opposition aux patrons tonaux $[\mathrm{B} \mathrm{B}+\mathrm{H}]$ et $[\mathrm{H} \mathrm{H}+\mathrm{B}]$ associés au niveau du mot prosodique. Il existe des variations dans la réalisation du SA. Le SA intégral comprend la courbe intonative en forme de chapeau en entier. Mais on trouve aussi, et souvent, des SA partiels qui ne comprennent que la chute finale. Il n'existe pas de SA partiel avec la montée seule. Cette observation suggère que la présence de la montée finale, dorénavant la courbe intonative de MPr-1 implique la présence de la descente en pénultième, i.e. la courbe intonative de MPr-2. Par contre, la courbe associée au MPr-2 peut exister en autonomie sans l'appui d'un MPr-1 à la courbe montante. Par conséquent la présence de la montée en MPr-1 est impliquée par MPr-2, mais MPr-2 peut se manifester en l'absence de tout autre patron tonal du même niveau hiérarchique. Lorsque le ton associé à la frontière finale du SA est bas (B-) il génère du downstep dans le SA qui suit, à savoir que la réalisation du sommet du deuxième SA se situe à un niveau inférieur au ton haut dans le premier SA. Ceci est illustré en Fig.1 et Fig.2. En revanche, la réalisation du downstep est bloquée si le ton final est haut (H-), et cette situation est représentée en Fig. 3. Enfin, des tons de marquage du syntagme intonatif se réalisent à la suite du dernier SA.

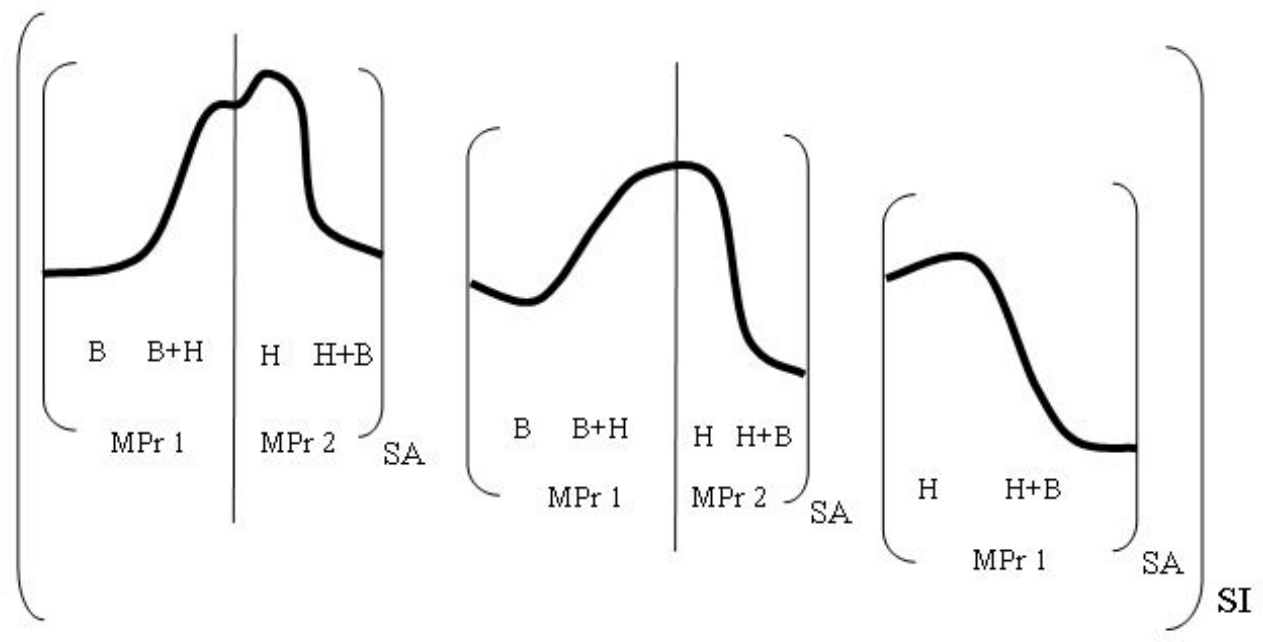

Fig. 1: Représentation schématique de l'intonation en créole guadeloupéen

Les tracés intonatifs en Fig. 2 et Fig. 3 illustrent ces faits. Fig. 2 correspond à l'énoncé « Lyne vomit le melon ». L'énoncé comporte trois MPr organisés en deux SA. Le premier SA reste intégral et il forme un chapeau. Le deuxième SA est partiel et il est constitué de la séquence $[\mathrm{H} \mathrm{H}+\mathrm{B}]$ dans laquelle la chute est associée à la pénultième syllabe du MPr, en gras melon la. De plus, comme la frontière entre les deux SA contient le ton $\mathrm{B}-$, le deuxième $\mathrm{SA}$ est réalisé à une fréquence moins élevée, dont le niveau des tons hauts 
correspond quasiment à la fréquence du ton bas qui précède. Les bulles indiquent la fréquence fondamentale au début de l'énoncé, puis le maximum et le minimum de chaque SA. La réalisation de downstep est formalisée par un point d'exclamation $(! \mathrm{H})$ qui signifie que le ton haut se réalise plus bas que dans le SA précédent. La réalisation de downstep dans ces modalités est très commune. L'énoncé en Fig. 3 est constitué de quatre MPr, groupés en deux SA chacun pleinement réalisé avec son patron intonatif en forme de chapeau, comme suit [mè la maye] [Alen e Lin], «Le maire a marié Alain et Lyne ». Le ton indiquant la fin du premier SA est $\mathrm{H}-$, ce qui bloque la réalisation de downstep. Ainsi les tons hauts demeurent-ils dans le même diapason de fréquence. La montée dans le premier MPr intervient toujours sur la dernière syllabe, mè $\boldsymbol{l a}$ et alen. La chute dans le deuxième MPr est toujours alignée avec la pénultième maye. Lin étant monosyllabique, la chute est associée à cette seule syllabe, mais plutôt avec le début de la syllabe, et non la fin.

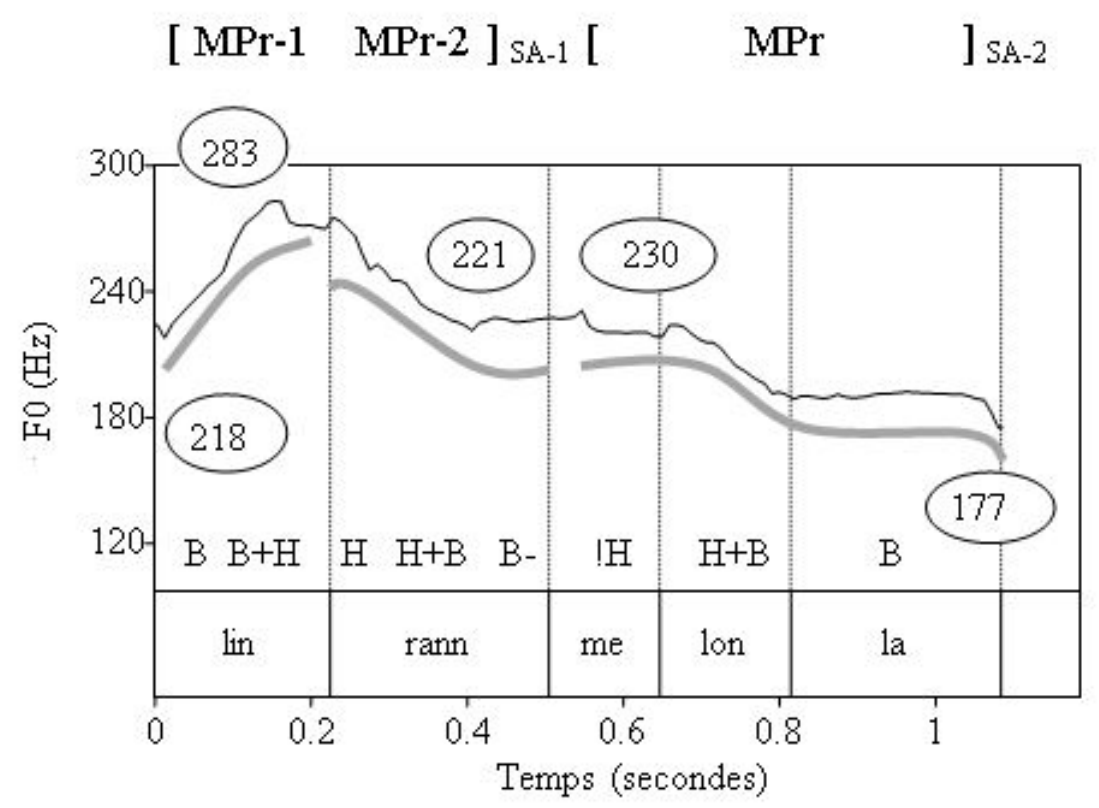

Fig. 2: Énoncé avec downstep, comportant trois mots prosodiques 


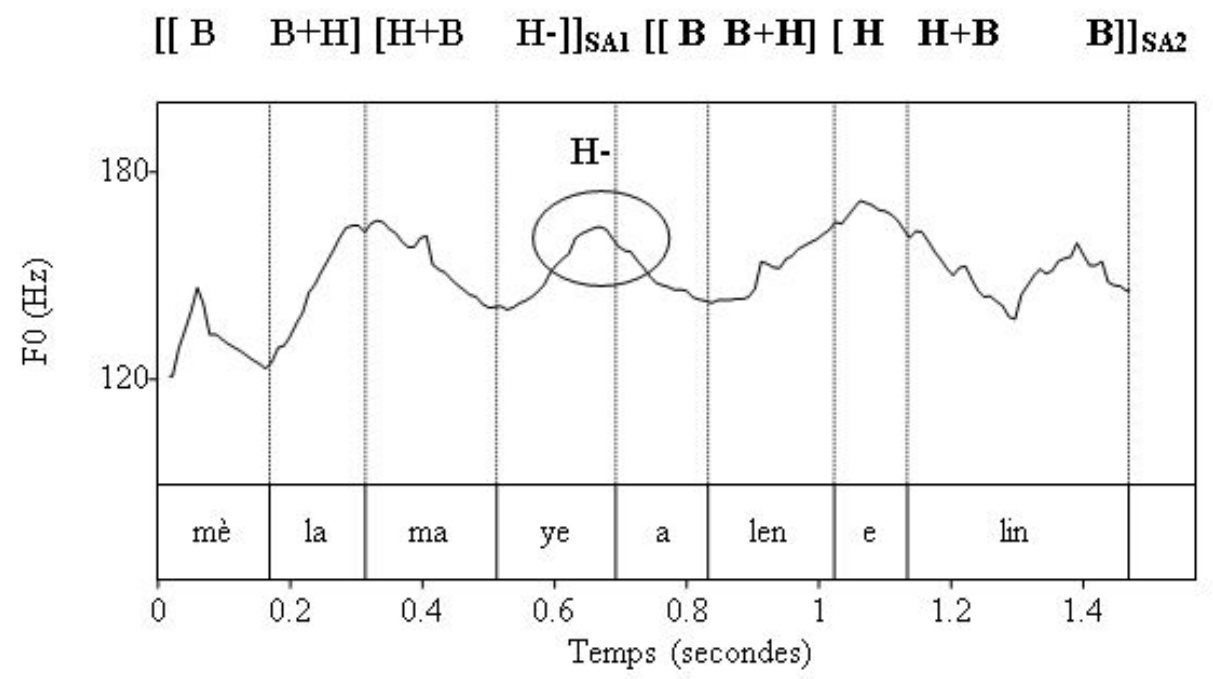

Fig. 3: Énoncé illustrant l'absence de downstep après un ton H- associé au SA

\section{Observations sur l'intonation en français antillais}

La courbe intonative en forme de chapeau du créole guadeloupéen est un trait unique des créoles à base lexicale française. Ce tracé intonatif ne semble pas provenir du français. Cependant, un certain nombre de ressemblances se retrouvent entre le créole et le français antillais. Deux caractéristiques du créole se retrouvent en français antillais. Les proéminences en pénultième syllabe omniprésentes en créole apparaissent également en français antillais. Ils se réalisent plutôt sous forme de proéminence associée à un ton $\mathrm{H}$ en début de mot. De même, la courbe en forme de butte se retrouve aussi en français antillais.

Un corpus trilingue de discours spontané, constitué de recettes antillaises a été enregistré auprès de métropolitaines locutrices du français hexagonal, vivant en Guadeloupe, ainsi qu'auprès de Guadeloupéennes qui ont donné les mêmes recettes en créole et en français antillais. Chaque femme a donné trois recettes, celle du court-bouillon, celle du colombo et une recette libre au choix. Ce corpus est très différent du précédent puisqu'il ne comporte que du discours spontané. Évidemment, les recettes comprennent du vocabulaire culinaire et quelques structures qui se ressemblent, du type faire revenir des oignons ou mettre sur le feu, mais chaque locutrice était libre d'utiliser son style et ses expressions favorites. L'examen des similarités et différences entre les tracés intonatifs dans les trois variétés linguistiques met en évidence quelques ressemblances entre le français antillais et le créole guadeloupéen. Les énoncés en français antillais révèlent que les débuts de mot, ou en tout cas la partie non-finale du mot, que ce soit la syllabe initiale ou la pénultième portent la proéminence mélodique dans certaines positions dans l'énoncé. Pour l'oreille du métropolitain il en résulte une illusion auditive que les Guadeloupéens insistent sur chaque mot, que leur discours est emphatique. En réalité il s'agit d'un plus grand nombre de mouvements de tons, plus de transitions, des alternances plus fréquentes entre tons haut et bas, réalisés dans un seul mot, et non pas à l'échelle du syntagme accentuel, comme en français standard. On constate en français antillais une grande fréquence d'emplois de chapeaux répartis à cheval entre deux mots qui s'organisent selon les mêmes modalités que les chapeaux en créole, à savoir une montée en syllabe finale dans un mot, suivi du maintien du ton haut au début du mot ou syntagme suivant, puis descente au milieu du mot.

Dans l'extrait de recette suivant, les parties en gras sont représentées dans Fig. 4 à Fig. 6. Le symbole «\|» représente le paroxysme de la montée et la ligne de partage entre les deux MPr. « Je mets de l'ail, je mets... des oignons, du girofle, très peu de poivre hein. J'utilise pas de poivre. Et donc je laisse || macérer (Fig. 4). Je mets aussi... un peu de-- cela peut être euh... du massalé. C'est- c'est la pâte à 
978-2-7598-0534-1, Paris, 2010, Institut de Linguistique Française

Phonétique, phonologie et interfaces

DOI $10.1051 / \mathrm{cmlf} / 2010249$

colombo que je mélange || un peu (Fig. 5) - un petit peu dedans - et je laisse $\|$ poser (Fig. 6). Et, à peu près 10 minutes, hein? » (Recette du colombo de JS).

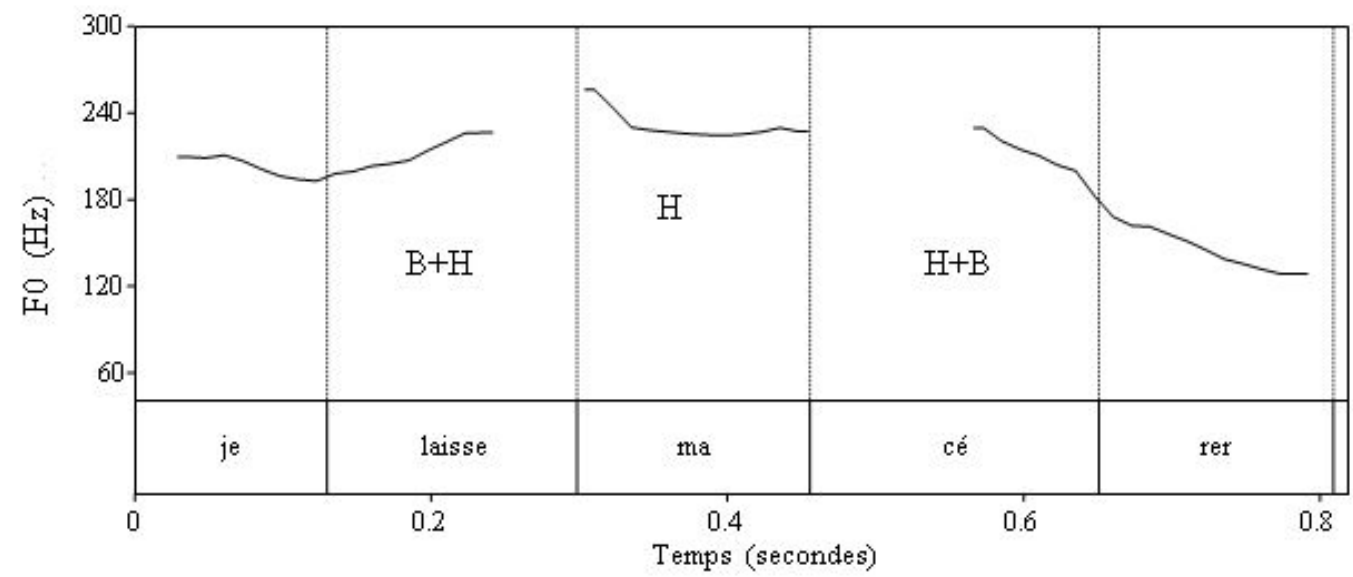

Fig. 4

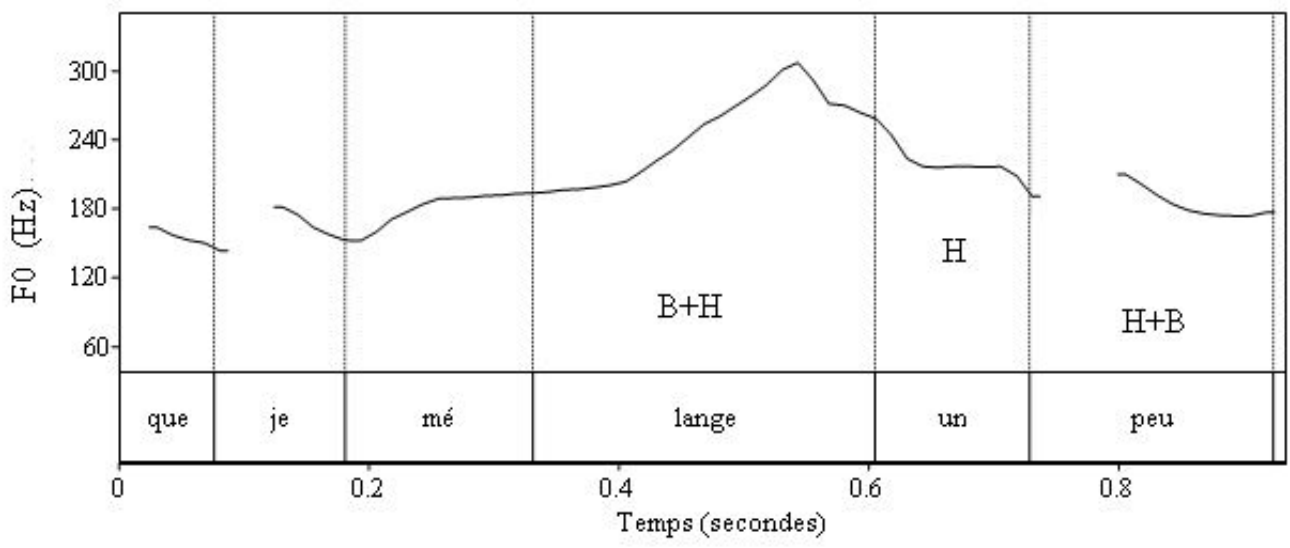

Fig. 5 


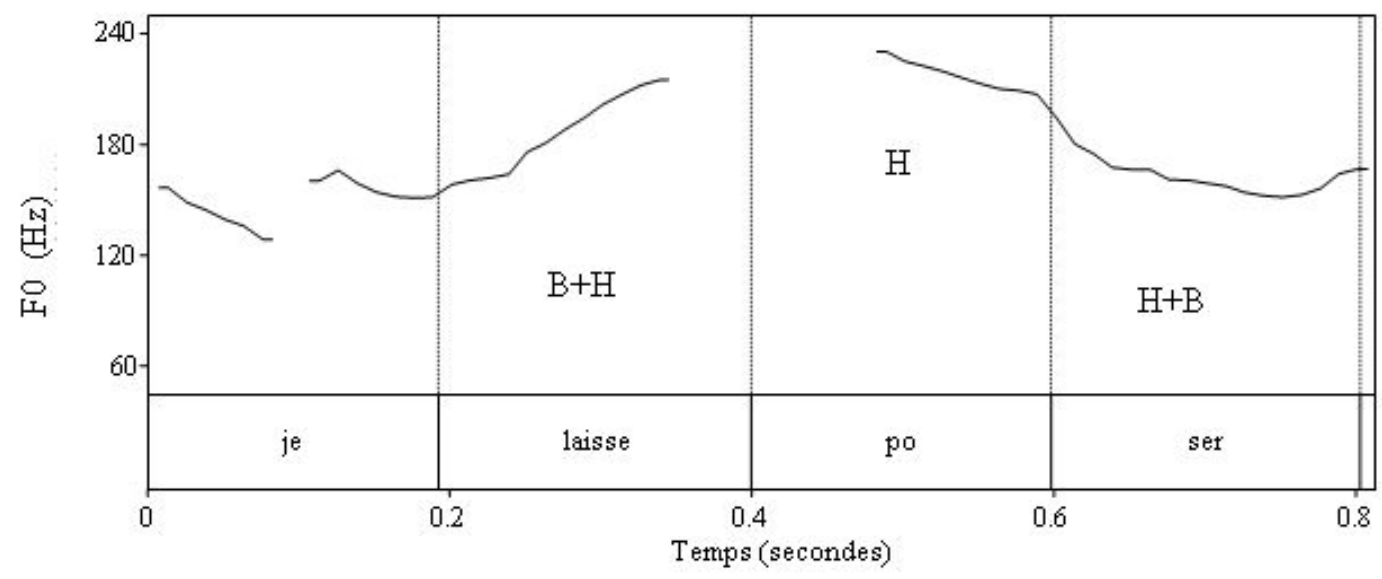

Fig. 6

\section{Conclusion}

L'hypothèse que le créole influence la structure du français antillais est soutenue par un certain nombre de traits linguistiques régionaux qu'on retrouve dans les deux langues. Que ce soit dans le domaine du lexique, de la syntaxe ou de la prosodie et la phonétique, il apparaît qu'il existe un fonds commun créoloantillais représentatif du contact de langues aux Antilles. Ce fonds commun est le résultat de la superposition du contact de langue qui fut à l'origine de la formation du créole d'une part, et du contact de langue contemporain entre créole et français. Dans le contact contemporain, le créole semble jouer deux grands rôles. D'une part, il participe au maintien des traits régionaux du français antillais, et d'autre part, il semble communiquer des traits au français antillais contemporain. Il est plus aisé de découvrir les traits provenant du créole lorsque l'on ne peut présumer une origine hexagonale de ces traits. Ainsi la construction factitive, qui à ma connaissance n'a pas d'équivalent dans l'Hexagone, appuie fortement l'hypothèse de l'emprunt au créole. La prononciation du [r] donne un exemple quant aux évolutions simultanées en créole et en français antillais.

Si l'on veut saisir la vraie nature du français antillais, il est important de s'atteler à sa description, pendant que la situation sociolinguistique qui est à l'origine de ce système est encore vivace. En effet les influences du créole sont certainement le produit non seulement du bilinguisme des Guadeloupéens, mais plus important, du fait que un très grand nombre de locuteurs furent jusqu'à une époque récente élevés dans des foyers monolingues. Ces locuteurs ont certainement transféré des traits dans le processus d'acquisition du français langue seconde.

\section{Références bibliographiques}

Beckman, Mary E., and Pierrehumbert, Janet B. 1986. Intonational structure in Japanese and English. Phonology Yearbook 3, 255-310.

Nespor, Marina, and Vogel, Irene. 1986. Prosodic Phonology. Dordrecht: Foris.

Pierrehumbert, Janet B. 1980. Phonology and Phonetics of English intonation, PhD Thesis, MIT.

Pierrehumbert, Janet Breckenridge, and Beckman, Mary E. 1988. Japanese tone structure. Cambridge, Mass. : MIT Press.

Selkirk, Elisabeth. 1980. The role of prosodic categories in English word stress. Linguistic Inquiry 11, 563-605. 
978-2-7598-0534-1, Paris, 2010, Institut de Linguistique Française

Phonétique, phonologie et interfaces

DOI $10.1051 / \mathrm{cmlf} / 2010249$

\section{Ressources en ligne:}

Ethnologue: www.ethnologue.org/show_country.asp?name=GP

INSEE. 2009. Recensement de la population. Populations légales en vigueur à compter du 1er janvier 2009. Sous la direction de Jean-Philippe Cotis.

www.insee.fr/fr/ppp/bases-de-donnees/recensement/populations-legales/pages2008/pdf/dep971.pdf

Joshua Project: www.joshuaproject.net/peopctry.php?rog3=GP\&rop3=103059 